\title{
Evaluation of Simplified Fluid Intake and Output Recording Schemes for the Self-management in Patients with Heart Failure
}

\author{
$\mathrm{Na} \operatorname{Lin}^{1}$, Xiaohuan $\mathrm{Chen}^{2}$, Xiufang Huang ${ }^{2}$, Donghui $\mathrm{Liu}^{2}$, Zhiyong $\mathrm{Wu}^{2}$, Yansong Guo ${ }^{2}$, \\ and Hong $\mathrm{Li}^{2}$ \\ ${ }^{1}$ Fujian Provincial Hospital \\ ${ }^{2}$ Affiliation not available
}

May 5, 2020

\begin{abstract}
Rationale, aims, and objectives: Fluid management plays a pivotal role for heart failure (HF) patients. Medical fluid intake and output recording scheme by health care professional is complicated, which is not easily conducive to carry out by HF patients for self-management at home. This study aimed to optimize the professional fluid records for the self-management of HF patients and evaluate the efficacy of this simplified recording scheme of fluid intake and output. Methods: A randomized, non-blinded and controlled trial with allocation concealment was conducted. A total of $140 \mathrm{HF}$ patients were enrolled and randomly divided into professional recording group (PRG, $\mathrm{n}=70$ ) and simplified recording group (SRG, $\mathrm{n}=70$ ). Ultimately, 129 HF patients (PRG, $n=65$, and SRG, $n=64$ ) completed these experiments. Basic clinical characteristics, days of clinical stability, clinical congestion score (CCS), Minnesota Living with Heart Failure Questionnaire (MLHFQ) and frequency of electrolyte disturbances in these patients were collected. Results: Compared to PRG patients, SRG patients also improved their HF symptoms (including shortness of breath and fluid retention), and did not show the prolonged hospitalization time after similar intravenous diuretic treatment. Additionally, the parameters of clinical stability, CCS, MLHFQ, electrolyte disturbances and body weight in SRG patients were not inferior to that of PRG patients $(\mathrm{P}>0.05)$. Conclusions: This simplified fluid intake and output recording scheme was safe, efficient and non-inferior to the professional mode, which might effectively enhance their feasibility of self-management, and improve their quality of life in HF patients.
\end{abstract}

\section{KEYWORDS:}

heart failure, nursing, self-management, quality of life, fluid intake and output

\section{ABBREVIATIONS:}

$\mathrm{AF}$ : atrial fibrillation;

ALT: alanine aminotransferase;

AMI: acute myocardial infarction;

AST: aspartate aminotransferase;

CCS: clinical congestion score;

CKD: chronic kidney diseases;

CRRT: continuous kidney substitution treatment;

DM: diabetes mellitus;

FBG: fasting blood glucose; 
HDL: high-density lipoprotein;

HF: heart failure;

IQR: interquartile range;

IV: intravenous injection;

LDL: low-density lipoprotein;

LOS: length of stay;

LVEF: left ventricular ejection fraction;

MLHFQ: Minnesota Living with Heart Failure Questionnaire;

NT-proBNP: N-terminal pro brain natriuretic peptide;

NYHA: New York Heart Association;

PRG: professional recording group;

QOL: quality of life;

RBC: red blood cell;

RDW: red cell distribution width;

SD: standard deviation;

SRG: simplified recording group;

TC: total cholesterol;

TCA: tricarboxylic acid;

UA: uric acid;

UN: urea nitrogen

\section{1 | INTRODUCTION}

Heart failure (HF) is a chronic and complicated syndrome ${ }^{1,2}$. Although HF treatment has developed, the long-term mortality improves poorly ${ }^{3-5}$. More than 1 million of HF patients are hospitalized each year, which almost occupy the top reason for elderly subjects admitted to hospital and account for more than $\$ 30$ billion of health care expenditure in USA ${ }^{6,7}$. HF decrease their quality of life (QOL), and effective treatment and care could remarkably alleviate their signs and discomforts ${ }^{8}$. Although fostering self-management skills in HF patients seems to be useless to reduce their mortalities, it could improve their QOL and decrease readmission rates $^{9}$. Surprisingly, the awareness rate of HF is much lower than that of expected in these patients, even those who have recurrent HF symptoms and follow the medical instructions for many years ${ }^{10,11}$. Meanwhile, most HF patients usually feel unprepared to manage their life styles at home ${ }^{8}$.

Fluid management plays a pivotal role in the self-care of HF patients, which could avoid the recurrent dyspnea symptoms ${ }^{1,2}$. However, an effective fluid management is a challenging task for HF patients owing to the dynamic fluid status. Evaluation of fluid situation in vivo include monitoring body weight, counting fluid intake and output as well as physical examination (e.g. jugular venous distention, hepatojugular reflux, lung rales and pedal edema). Physical examination usually need the assistance of health care professionals ${ }^{1,2,12}$. While either in hospital or at home, monitoring body weight and recording fluid changes of intake and output remain two basic issues, especially after HF patients return to their daily life. In fact, it is controversial to regard body weight as a major indicator for fluid evaluation. Because body weight is often affected by many factors, including clothes, diet, testing time, and ambient temperature ${ }^{13}$. Additionally, monitoring body weight sometimes is not easy to be performed, especially in those patients who are bedridden for years. 
Although many clinic guidelines for HF recommend that these patients should record their body weight every day, almost few acute HF accidences are forecasted through monitoring body weight due to the lower sensitivity of weight gain $(9 \%)^{14}$.

In addition, there are great differences in the diet compositions and habits between Western and Eastern subjects, causing an inconsistent understanding for water contained in foods. In Western countries, besides common solid and liquid foods, people often consume much semi-solid foods (e.g. purees and gelatin) which contain much water, and fluid in these foods is often calculated and counted ${ }^{13}$. In China, water in solid foods is also calculated and converted to fluid intake based on the moisture scales of foods following the professional fluid intake recoding schemes ${ }^{15}$. Additionally, it is awkward that the feces should always be considered. And the differences of ingredients and cooking methods, the irregularity of cognition in HF patients and their family members, and the tedious mode of professional recording scheme could all cause the poor compliance of monitoring fluid intake and output ${ }^{16}$. Therefore, whether the professional recording scheme could be simplified more easily for the self-management of HF patients at home, should be reconsidered.

In this study, a simplified recording scheme of fluid intake and output for HF patients was developed, which was safe, efficient and non-inferior to the professional mode in clinical stability, electrolyte imbalances and cardiac functions. This modified fluid recording mode, as an effective supplement to body weight for fluid self-management, might improve their QOL and reduce their recurrent hospitalization times for HF patients.

\section{2 | METHOD}

\section{1 | Study design}

Using a blinded end-point adjudication, a single-center randomized controlled trial (RCT) was conducted to evaluate the efficiency between the simplified and professional intake and output recording schemes.

\section{2 | Setting and subjects}

This study was carried out from October 1, 2018 to April 30, 2019 in the department of Cardiology of a tertiary first-class comprehensive hospital in southeastern China. All participants were completely informed the content of the consent form with signatures. The inclusion criteria were (i) age [?]18 years, (ii) diagnosis of HF according to 2017 ACC/AHA/HFSA Heart Failure Focused Update Guidelines with combination of clinical symptoms, physical examination, chest X-ray and echocardiography ${ }^{12}$, (iii) New York Heart Association (NYHA) class III-IV or Killip class II-IV, and (iv) daily fluid intake and output records following doctors' advices. The exclusion criteria consisted of hemodialysis, peritoneal dialysis, bedside ultrafiltration, continuous kidney substitution treatment (CRRT), cancer and another uncooperative disease (e.g. dementia and cognitive impairment).

\section{3 | Sampling}

Sample size was calculated basing on 2-sample equivalence model. The minimum sample size was calculated as 100 participants according to the significant level of $\alpha=0.05$, the statistical power of $80 \%$, the mean difference less than 1.3 days to clinical stability (primary outcome) between two groups, and the standard deviation of 2 days in each group from pretest ${ }^{17}$. Given an estimation drop-out rate (28\%), including in-hospital mortality rates and the ward conversion rates, a total of $140 \mathrm{HF}$ patients were enrolled.

\subsection{Randomization and allocation concealment}

After signing an informed consent and undergoing a basic assessment, HF patients were randomly assigned by using a random number table through an online random tool (https://tools.medsci.cn/rand/getNumWithCode) (random number seed: 70472134). Researcher No.1 was responsible for the randomization process, but not participating in the enrollment. Enrollment was performed by investigator No.2. In order to avoid mutual contamination among HF patients in the same ward, the potential patients in the same wards were also excluded. One hundred and forty patients were randomized and assigned to the professional recording group (PRG) or the simplified recording group 
(SRG). Eleven patients were excluded lately because of losing observation, in-hospital death, or transferring to other departments. The flow chart of patients screening and allocating was shown in Figure 1.

\section{5 | Intervention}

PRG patients proceeded the standard recording scheme of fluid intake and output according to the nursing textbook ${ }^{15}$. The intake fluid included oral fluid, "embedded water" in foods, "generated water" from food metabolism, intravenous infusion and blood transfusion. The output fluid included urine, feces, insensible losses (through skin and lung), drainage liquid, vomit, hemoptysis and sputum, bleeding, and wound drainage. SRG patients carried on a simplified record which optimized the fluid embedded in or generated from foods and those in feces as shown in Table 1.

Liquid foods were measured by using a container with "ml" capacity scale. Non-liquid foods were weighed by using a uniform electronic scale and recorded as "g". Especially, PRG patients should calculate the content of water according to food moisture conversion table ${ }^{15}$. Urine was measured as "ml" by a graduated cylinder, and watery stools were weighed. For those patients with poor education or memory, the procedures were completed with the help of their family members or nurses.

\section{6 | Outcome measurements}

The primary outcome was the days from admission to clinical stability, which was defined by a decrease of 2 points of clinical congestion score (CCS) and the cessation of all intravenous pharmacotherapy (e.g. diuretics, inotropes or vasodilators) ${ }^{17-19}$. CCS is an instrument composed of 7 questions, which are designed to assess the congestion degrees in HF patients ${ }^{20}$. The score ranges from 1 to 22 points, and the higher scores imply the worse congestion degrees. Clinical stability was independently judged by investigator No.3 and cardiologist Dr.1 every day. If the judgment was not inconsistent, it should be determined by cardiologist Dr. 2. The group allocation was blinded to all judgers.

The other end points included the electrolyte disturbances, N-terminal pro brain natriuretic peptide (NTproBNP) levels. The most common electrolyte disturbances are high or low levels of serum sodium and potassium ${ }^{21,22}$. In this study, they were defined as: hyponatremia (serum sodium level $<135.00 \mathrm{mmol} / \mathrm{l}$ ), hypernatremia (serum sodium level $>145.00 \mathrm{mmol} / \mathrm{l}$ ), hypokalemia (serum potassium level $<3.50 \mathrm{mmol} / \mathrm{l}$ ), and hyperkalemia (serum potassium level $>5.00 \mathrm{mmol} / \mathrm{l})^{23}$. In addition, body weight was recorded after 24 hours since ceasing intravenous pharmacotherapy. Minnesota Living with Heart Failure Questionnaire (MLHFQ) was tested before leaving hospital. All above were in the charge of researcher No. 3.

\section{7 | Statistical analysis}

Data were expressed as mean $\pm \mathrm{SD}$ for normally distributed variables or as median [IQR] for skewed variables. The differences between groups were assessed by using independent Student's $t$ test (for normally distributed variables) or Mann-Whitney test (for skewed variables). Percentages were used to summarize the categorical variables. Pearson $x^{2}$ test was used to analyze the differences between groups for categorical variables. As a non-inferiority trial, all analyses were performed by per-protocol sets. The significant level was set at $P$ $<0.05$. All analyses were performed by using statistical program IBM SPSS software (Version 25.0).

\section{3 | RESULTS}

\section{1 | Clinical characteristics}

The clinical characteristics for PRG $(n=65)$ and SRG $(n=64)$ were presented in Table 2. There was no significant difference in body weight, hemoglobin, red blood cell (RBC), hematocrit, red cell distribution width (RDW), albumin, osmotic pressure, NT-proBNP, classification of cardiac function, left ventricular ejection fraction (LVEF), complications, use of intravenous diuretics, and the completeness of intake and output records $(P$ i.0.05). Meanwhile, hospitalization days and CCS accessed at admission in both groups were also similar respectively $(P$ ¿0.05).

\section{2 | Comparison of outcomes}


Compared to PRG patients, SRG patients also improved HF symptoms (e.g. shortness of breath and peripheral fluid retention) in the similar treatment duration after using intravenous diuretics. Shortness of breath and peripheral fluid retention are two important items in $\mathrm{CCS}^{20}$. Collectively, CCS between two groups also showed no statistical differences (SRG, 3.89+-0.99 vs. PRG, 4.19+-1.10; $P=0.110$ ). In addition, there were no significant differences of clinical stability in the days from admission to improvement between two groups. Furthermore, SRG patients also did not show the prolonged hospitalization time and the increased MLHFQ score. Therefore, both groups exhibited the similar improvement in HF symptoms and clinical signs. Besides, there was no statistical significance in the incidence of hypokalemia, hyperkalemia, hyponatremia, and hypernatremia as reported in Table $3(P$ i0.05).

\section{4 | DISSCUSSION}

$\mathrm{HF}$ is a terminal outcome of many cardiovascular diseases ${ }^{2}$. Effective fluid management is a principal way for HF treatment and care ${ }^{24}$. Body weight, urine volume and net fluid balance are considered to reflect the dynamic changes of fluid in vivo ${ }^{1,2,12}$. However, it is a hard challenge to obtain an accurate net fluid output and a series of body weight changes both in hospital and at home every day ${ }^{14,25}$. In this study, we simplified the fluid intake and output recording scheme that was much easier than the professional mode for the self-management of HF patients, and it was also efficient to monitor clinical stability and electrolyte balances. Therefore, this simplified fluid recording scheme might effectively help HF patients improve their QOL, reduce their rehospitalization times, and especially enhance the feasibility of self-management.

Given the decreased activities of endurance and the difficulties of changing positions from recumbent to standing, monitoring weight daily is often rejected by HF patients. In addition, the accuracy of body weight measurement was also interfered by many factors ${ }^{26}$. Furthermore, as one of the common markers to assess congestion, daily weight loss has no direct relationships with fluid loss and symptom improvement ${ }^{27,28}$. Thus, only monitoring body weight hardly reflect the daily fluid balance.

Measuring fluid intake and output has long been supposed to be precise and normative. However, it is hard to carry out owing to the low self-management abilities and the complexities of counting fluid intake and output for HF patients ${ }^{16}$. These patients often forget to record fluid intake, reduce the cooperation to collect urine and stool, and ignore to count the fluid contained in foods (such as fruits and vegetables) 29. In many cases, HF patients are educated to regularly measure fluid intake and output following the textbook disciplines. However, these patients often encounter the changes of lifestyle and physiological state, including bedridden with weakness, urea incontinence and so on. Therefore, this study simplified the professional recording scheme, defining fluid intake and output as net fluid volume. In contrast to the professional mode, it is easier to be manipulated and followed by HF patients. And it did not change the clinical stability and increase the disorders of electrolyte in HF patients. The body weight, NT-proBNP and MLHFQ also presented no significant difference $(P>0.05)$. Some studies also showed that non-dogmatic recording schemes does not cause other adverse results ${ }^{30}$.

Researchers recommended that severe HF patients should moderately restrict fluid, including no more than intaking 1,500-2,000 $\mathrm{ml}$ of water, and over $500 \mathrm{ml}$ of additional net output every day ${ }^{1,9,12}$. Generally, healthy subjects intake fluid about average $1,500 \mathrm{ml} /$ day in normal conditions. However, the body actually need about $2,500 \mathrm{ml}$ of water to maintain the physical functions. The additional $1,000 \mathrm{ml}$ of water is acquired from "embedded water" $(150 \mathrm{ml})$ contained in foods and "generated water" $(750 \mathrm{ml})$ from food metabolism which is produced from tricarboxylic acid (TCA) cycle and oxidative phosphorylation processes (mainly carbohydrate and fatty acids) ${ }^{13}$. The fluid output include: urine about 1,400-1,500 ml/day, feces about 100-200 ml/day, and insensible loss about 800-900 ml/day (e.g. perspiration and evaporation through skin, and water vapor expired to air through lungs) ${ }^{13}$. Coincidentally, the content of water in food is close to that of feces plus insensible loss from skin and lungs. Therefore, when we monitor fluid intake and output in HF patients, we could almost ignore the fluid intake from foods and the fluid output from feces and insensible loss.

Frankly, there are several limitations in the present study. First, this study was performed in a single center, 
and a hospital-specific bias could not be excluded. Second, this study just observed the changes of HF patients in hospital, but not at home. We hope SRG patients could also adaptively record their net fluid intake and output as a part of his/her daily lifestyle every day. Third, more importantly, we wonder whether this simplified fluid intake and output records together with monitoring body weight could really improve QOL and reduce hospitalization times in these patients for a long time. And we are proceeding a follow-up procedure now.

\section{5 | CONCLUSION}

This study found that the simplified fluid intake and output recording scheme was safe, efficient and noninferior to the professional mode in clinical stability, electrolyte balance, body weight and NT- proBNP levels. We expected that the simplified fluid records combined with monitoring body weight might effectively help HF patients enhance self-management, improve QOL and reduce rehospitalization times.

\section{AUTHOR CONTRIBUTION}

$\mathrm{Na}$ Lin conceived the study and wrote the manuscript. Xiaohuan Chen was responsible for data management and study design. Xiufang Huang, Donghui Liu, and Zhiyong Wu supported several experiments and analyzed the data. Yansong Guo and Hong Li supervised the research and revised the manuscript. All authors read and approved the final manuscript.

\section{CONFLICT OF INTEREST}

The authors confirm that there are no conflicts of interest.

\section{ETHICAL APPROVAL}

The study was approved by the ethics committee of Fujian Provincial Hospital (No: K2018-09-004), and registered in Chinese Clinical Trials Registry (Identification Number: ChiCTR1800018523). The study was conducted according to Helsinki Declaration, information about the application has been made and signed.

\section{INFORMED CONSENT}

Informed consent was obtained from all individual participants included in the studies.

\section{ACKNOWLEDGEMENTS}

This project was supported by Grant 81571379 and 81873495 from the National Natural Science Foundation of China and Grant 2017XQ1142 from the Startup Funds for Scientific Research of Fujian Medical University, China.

\section{REFERENCES}

1. Yancy CW, Jessup M, Bozkurt B, et al. 2013 ACCF/AHA guideline for the management of heart failure: a report of the American College of Cardiology Foundation/American Heart Association Task Force on Practice Guidelines. J Am Coll Cardiol. 2013;62(16):e147-239.https://doi.org/10.1161/CIR.0b013e31829e87r6

2. Ponikowski P, Voors AA, Anker SD, et al. 2016 ESC Guidelines for the diagnosis and treatment of acute and chronic heart failure: The Task Force for the diagnosis and treatment of acute and chronic heart failure of the European Society of Cardiology (ESC)Developed with the special contribution of the Heart Failure Association (HFA) of the ESC.Eur Heart J. 2016;37(27):2129-2200.https://doi.org/10.1093/eurheartj/ehw128

3. Betihavas V, Frost SA, Newton PJ, et al. An Absolute Risk Prediction Model to Determine Unplanned Cardiovascular Readmissions for Adults with Chronic Heart Failure. Heart Lung Circ. 2015;24(11):10681073.https://doi.org/10.1016/j.hlc.2015.04.168

4. Kalter-Leibovici O, Freimark D, Freedman LS, et al. Disease management in the treatment of patients with chronic heart failure who have universal access to health care: a randomized controlled trial.BMC Med. 2017;15(1):90.https://doi.org/10.1186/s12916-017-0855-z 
5. Koehler F, Koehler K, Deckwart O, et al. Telemedical Interventional Management in Heart Failure II (TIM-HF2), a randomised, controlled trial investigating the impact of telemedicine on unplanned cardiovascular hospitalisations and mortality in heart failure patients: study design and description of the intervention. Eur J Heart Fail.2018;20(10):1485-1493.https://doi.org/10.1002/ejhf.1300

6. Heidenreich PA, Albert NM, Allen LA, et al. Forecasting the impact of heart failure in the United States: a policy statement from the American Heart Association. Circ Heart Fail. 2013;6(3):606619.https://doi.org/10.1161/HHF.0b013e318291329a

7. Zhang Y, Zhang J, Butler J, et al. Contemporary Epidemiology, Management, and Outcomes of Patients Hospitalized for Heart Failure in China: Results From the China Heart Failure (China-HF) Registry. J Card Fail. 2017;23(12):868-875.https://doi.org/10.1016/j.cardfail.201\%.09.014

8. Jiang Y, Shorey S, Nguyen HD, et al. The development and pilot study of a nurse-led HOMe-based HEart failure self-Management Programme (the HOM-HEMP) for patients with chronic heart failure, following Medical Research Council guidelines. Eur $J$ Cardiovasc Nurs.2019:1474515119872853.https://doi.org/10.1177/1474515119872853

9. Sherwood A, Blumenthal JA, Koch GG, et al. Effects of Coping Skills Training on Quality of Life, Disease Biomarkers, and Clinical Outcomes in Patients With Heart Failure: A Randomized Clinical Trial. Circ Heart Fail. 2017;10(1).https://doi.org/10.1161/CIRCHEARTFAILURE.116.003410

10. van der Wal MH, Jaarsma T, Moser DK, van Gilst WH, van Veldhuisen DJ. Qualitative examination of compliance in heart failure patients in The Netherlands. Heart Lung. 2010;39(2):121130.https://doi.org/10.1016/j.hrtlng.2009.07.008

11. Dinh H, Bonner A, Ramsbotham J, Clark R. Self-management intervention using teach-back for people with heart failure in Vietnam: A cluster randomized controlled trial protocol. Nurs Health Sci.2018;20(4):458-463.https://doi.org/10.1111/nhs.12534

12. Yancy CW, Jessup M, Bozkurt B, et al. 2017 ACC/AHA/HFSA Focused Update of the 2013 ACCF/AHA Guideline for the Management of Heart Failure: A Report of the American College of Cardiology/American Heart Association Task Force on Clinical Practice Guidelines and the Heart Failure Society of America. Circulation. 2017;136(6):e137-e161.https://doi.org/10.1161/CIR.0000000000000509

13. Berman A, Snyder S, Frandsen G. Kozier \& Erb's Fundamentals of Nursing Concepts, Process, and Practice In: TENTH ed.: Julie Levin Alexander; 2016:1329,1330 p.

14. Gudmundsson K, Lynga P, Rosenqvist M, Braunschweig F. Monitoring of Daily Body Weight and Intrathoracic Impedance in Heart Failure Patients With a High Risk of Volume Overload Decompensation. Clin Cardiol.2016;39(8):446-452.https://doi.org/10.1002/clc.22547

15. Li XH, Shang SM. Fundamentals of Nursing. In: SIXTH ed.: People's Medical Publishing House; 2017:215,216 p. (in Chinese)

16. Halmo R, Galuszka J, Langova K, Galuszkova D. Self care in patients with chronic heart failure. Pilot study - self care includes problems.Biomedical Papers. 2015;159(1):124130.https://doi.org/10.5507/bp.2013.044

17. Travers B, O'Loughlin C, Murphy NF, et al. Fluid restriction in the management of decompensated heart failure: no impact on time to clinical stability. $J$ Card Fail. 2007;13(2):128132.https://doi.org/10.1016/j.cardfail.2006.10.012

18. Aliti GB, Rabelo ER, Clausell N, Rohde LE, Biolo A, Beck-da-Silva L. Aggressive fluid and sodium restriction in acute decompensated heart failure: a randomized clinical trial. JAMA Intern Med.2013;173(12):1058-1064.https://doi.org/10.1001/jamainternmed.2013.552 
19. Feijo MK, Biolo A, Ruschel KB, Orlandin L, Aliti GB, Rabelo-Silva ER. Effect of a diuretic adjustment algorithm and nonpharmacologic management in patients with heart failure: study protocol for a randomized controlled trial. Trials. 2015;16:44.https://doi.org/10.1186/s13063-015-0559-7

20. Sauer J, Rabelo ER, Castro RA, et al. Nurses' performance in classifying heart failure patients based on physical exam: comparison with cardiologist's physical exam and levels of N-terminal pro-B-type natriuretic peptide. J Clin Nurs. 2010;19(23-24):3381-3389.https://doi.org/10.1111/j.1365-2702.2010.03403.x

21. Polcwiartek C, Hansen SM, Kragholm K, et al. Prognostic role of serum sodium levels across different serum potassium levels in heart failure patients: A Danish register-based cohort study. Int $J$ Cardiol. 2018;272:244-249.https://doi.org/10.1016/j.ijcard.2018.08.045

22. Imran TF, Kurgansky KE, Patel YR, et al. Serial sodium values and adverse outcomes in heart failure with preserved ejection fraction.Int $J$ Cardiol. 2019;290:119124.https://doi.org/10.1016/j.ijcard.2019.03.040

23. Jantzen D, Felver L. Canadian Fundamentals of Nursing. In: SIXTH ed.: ELSEVIER; 2019:3244,3245 p.

24. Bai L, Xu DL, Zhang YH, et al. Expert recommendation on the voume control in heart failure management. Chin J Heart Fail \& Cardiomyopathy. 2018;2:9. (in Chinese)

25. Abraham WT, Compton S, Haas G, et al. Intrathoracic impedance vs daily weight monitoring for predicting worsening heart failure events: results of the Fluid Accumulation Status Trial (FAST). Congest Heart Fail. 2011;17(2):51-55.https://doi.org/10.1111/j.1751-7133.2011.00220.x

26. Ambrosy AP, Cerbin LP, Armstrong PW, et al. Body Weight Change During and After Hospitalization for Acute Heart Failure: Patient Characteristics, Markers of Congestion, and Outcomes: Findings From the ASCEND-HF Trial. JACC Heart Fail. 2017;5(1):1-13.https://doi.org/10.1016/j.jchf.2016.09.012

27. Kociol RD, McNulty SE, Hernandez AF, et al. Markers of decongestion, dyspnea relief, and clinical outcomes among patients hospitalized with acute heart failure. Circ Heart Fail. 2013;6(2):240245.https://doi.org/10.1161/CIRCHEARTFAILURE.112.969246

28. Miller WL, Mullan BP. Understanding the heterogeneity in volume overload and fluid distribution in decompensated heart failure is key to optimal volume management: role for blood volume quantitation. JACC Heart Fail. 2014;2(3):298-305.https://doi.org/10.1016/j.cnur.2017.01.003

29. Testani JM, Brisco MA, Kociol RD, et al. Substantial Discrepancy Between Fluid and Weight Loss During Acute Decompensated Heart Failure Treatment. Am J Med. 2015;128(7):776-783 e774.https://doi.org/10.1016/j.amjmed.2014.12.020

30. Miller RK, Thornton N. Does Evidence Drive Fluid Volume Restriction in Chronic Heart Failure? Nurs Clin North Am. 2017;52(2):261-267.https://doi.org/10.1016/j.jchf.2014.02.007

\section{FIGURE LEGEND}

\section{FIGURE 1. Flow chart of patients screening and allocating.}

\section{Hosted file}

Figure 1_2020_lin.pptx available at https://authorea.com/users/293322/articles/421252evaluation-of-simplified-fluid-intake-and-output-recording-schemes-for-the-self-

management-in-patients-with-heart-failure

\section{Hosted file}

20200118_JECP_table.docx available at https://authorea.com/users/293322/articles/421252evaluation-of-simplified-fluid-intake-and-output-recording-schemes-for-the-selfmanagement-in-patients-with-heart-failure 